\title{
Weak type estimates associated to Burkholder's martingale inequality
}

Javier Parcet

\begin{abstract}
Given a probability space $(\Omega, A, \mu)$, let $A_{1}, A_{2}, \ldots$ be a filtration of $\sigma$-subalgebras of $\mathrm{A}$ and let $\mathrm{E}_{1}, \mathrm{E}_{2}, \ldots$ denote the corresponding family of conditional expectations. Given a martingale $f=\left(f_{1}, f_{2}, \ldots\right)$ adapted to this filtration and bounded in $L_{p}(\Omega)$ for some $2 \leq p<\infty$, Burkholder's inequality claims that

$$
\|f\|_{p} \sim_{\mathrm{c}_{p}}\left\|\left(\sum_{k=1}^{\infty} \mathrm{E}_{k-1}\left(\left|d f_{k}\right|^{2}\right)\right)^{\frac{1}{2}}\right\|_{p}+\left(\sum_{k=1}^{\infty}\left\|d f_{k}\right\|_{p}^{p}\right)^{\frac{1}{p}} .
$$

Motivated by quantum probability, Junge and $\mathrm{Xu}$ recently extended this result to the range $1<p<2$. In this paper we study Burkholder's inequality for $p=1$, for which the techniques must be different. Quite surprisingly, we obtain two non-equivalent estimates which play the role of the weak type $(1,1)$ analog of Burkholder's inequality. As application we obtain new properties of Davis decomposition for martingales.
\end{abstract}

\section{Introduction and Main Results}

Sums of independent random variables and martingale inequalities are nowadays powerful tools in classical harmonic analysis. Mostly in the 70's and 80's, the works of Bourgain, Burkholder, Davis, Gundy, Pisier, Rosenthal and many others illustrated a fruitful interaction between these subjects and Calderón-Zygmund theory of singular integrals as well as LittlewoodPaley theory. Our first motivation in this paper comes from a fundamental result due to Burkholder $[1,2]$ which can be stated as follows. Given a probability space $(\Omega, \mathrm{A}, \mu)$, let $\mathrm{A}_{1}, \mathrm{~A}_{2}, \ldots$ be a filtration of $\sigma$-subalgebras of $\mathrm{A}$

2000 Mathematics Subject Classification: 42B25, 60G46, 60G50.

Keywords: Burkholder martingale inequality, Davis and Gundy decompositions. 
and let $E_{1}, E_{2}, \ldots$ denote the corresponding family of conditional expectations. Given $2 \leq p<\infty$ and a martingale $f=\left(f_{1}, f_{2}, \ldots\right)$ adapted to this filtration and bounded in $L_{p}(\Omega)$, we have

$$
\|f\|_{p} \sim_{c_{p}}\left\|\left(\sum_{k=1}^{\infty} \mathrm{E}_{k-1}\left(\left|d f_{k}\right|^{2}\right)\right)^{\frac{1}{2}}\right\|_{p}+\left\|\left(\sum_{k=1}^{\infty}\left|d f_{k}\right|^{p}\right)^{\frac{1}{p}}\right\|_{p} .
$$

The first term on the right is called the conditional square function of $f$, while the second is the $p$-variation of $f$. The optimal growth of the equivalence constant is given by $\mathrm{c}_{p} \sim p / \log p$ as $p \rightarrow \infty$ and $\left(\mathrm{B}_{p}^{+}\right)$fails on $L_{\infty}(\Omega)$, we refer to the papers $[8,9]$ for more details. Apart from the relation with harmonic analysis, Burkholder's inequality has deep implications in the geometry of Banach spaces. Let us mention for instance Maurey/Pisier's theory of type and cotype or the isomorphism and embedding theory of $L_{p}$ spaces via $p$-stable processes.

These assertions are justified by the following observations. First, Rosenthal's inequality [21] appears as the particular case where the sequence $d f_{1}, d f_{2}, \ldots$ is given by a family of independent mean-zero random variables $d f_{k}=\xi_{k}$. In this case we have $\mathrm{E}_{k-1}\left(\left|d f_{k}\right|^{2}\right)=\left\|\xi_{k}\right\|_{2}^{2}$ and deduce Rosenthal's inequality

$$
\left\|\sum_{k=1}^{\infty} \xi_{k}\right\|_{p} \sim_{\mathrm{c}_{p}}\left(\sum_{k=1}^{\infty}\left\|\xi_{k}\right\|_{2}^{2}\right)^{\frac{1}{2}}+\left(\sum_{k=1}^{\infty}\left\|\xi_{k}\right\|_{p}^{p}\right)^{\frac{1}{p}} .
$$

Moreover, we can go further and take $\xi_{k}=\lambda_{k} \varepsilon_{k}$ with $\lambda_{k} \in \mathbb{C}$ and $\varepsilon_{1}, \varepsilon_{2}, \varepsilon_{3}, \ldots$ independent Bernoulli random variables equidistributed in \pm 1 , the reader can think for instance in the sequence of Rademacher functions on the unit interval. In this case, the two terms on the right of $\left(\mathrm{R}_{p}\right)$ collapse into the first one and we recover the classical Khintchine inequalities for $2 \leq p<\infty$

$$
\left\|\sum_{k=1}^{\infty} \lambda_{k} \varepsilon_{k}\right\|_{p} \sim_{\mathrm{c}_{p}}\left(\sum_{k=1}^{\infty}\left|\lambda_{k}\right|^{2}\right)^{\frac{1}{2}}
$$

Our second motivation comes from the noncommutative analogues of the results mentioned so far. Roughly speaking, we replace functions by operators (this process is known as quantization) and study noncommutative generalizations of the classical results. In this setting, the main objects are noncommutative $L_{p}$ spaces constructed over von Neumann algebras [17] and noncommutative martingales [23]. The theory of noncommutative martingale inequalities (a subfield of quantum probability) has reached an spectacular development after Pisier/Xu's seminal paper [16]. Indeed, it can be said that almost every classical martingale inequality has been successfully transferred to the noncommutative setting. We find noncommutative analogues 
of the Burkholder-Gundy inequalities [16], Doob's maximal inequality [10] and weak type $(1,1)$ estimates for martingale transforms [18]. Burkholder's inequality was finally obtained by Junge and $\mathrm{Xu}$ in [12].

The new insight provided by the noncommutative formulation led Junge and $\mathrm{Xu}$ to extend in [12] Burkholder's inequality to the range $1<p \leq 2$. This extension was new even in the commutative case and can be explained (we only consider here commutative random variables) as follows. The right hand side of $\left(\mathrm{B}_{p}^{+}\right)$can be understood as the norm in the intersection of two Banach spaces, respectively called conditional and diagonal Hardy spaces of martingales. Thus, it is natural to guess that in the dual formulation of $\left(\mathrm{B}_{p}^{+}\right)$, we will find a sum of the dual Hardy spaces. Then, recalling the definition of the norm on a sum of Banach spaces, Burkholder's inequality for $1<p \leq 2$ reads as follows

$$
\left(\mathrm{B}_{p}^{-}\right) \quad\|f\|_{p} \sim_{\mathrm{c}_{p}} \inf _{f=g+h}\left\{\left\|\left(\sum_{k=1}^{\infty} \mathrm{E}_{k-1}\left(\left|d g_{k}\right|^{2}\right)\right)^{\frac{1}{2}}\right\|_{p}+\left\|\left(\sum_{k=1}^{\infty}\left|d h_{k}\right|^{p}\right)^{\frac{1}{p}}\right\|_{p}\right\}
$$

where the infimum runs over all possible decompositions of $f$ as a sum $f=g+h$ of two martingales. Note that the right expressions for $\left(\mathrm{B}_{2}^{+}\right)$and $\left(\mathrm{B}_{2}^{-}\right)$are equivalent so that the quadratic case explains the transition from intersections to sums. Let us mention that this is a typical phenomenon in the noncommutative setting which also appears for instance in the noncommutative Khintchine inequalities [14] or the noncommutative Burkholder-Gundy inequalities [16]. In particular, Junge/Xu's paper [12] illustrated how a noncommutative problem can give some light in its commutative counterpart! We think this is also the case in this paper.

The problem of determining the behavior of Burkholder's inequality on $L_{1}(\Omega)$ naturally came out after Junge/Xu's extension. As it was pointed out in [12], the upper estimate holds with an absolute constant c

$$
\|f\|_{1} \leq \mathrm{c} \inf _{f=g+h}\left\{\left\|\left(\sum_{k=1}^{\infty} \mathrm{E}_{k-1}\left(\left|d g_{k}\right|^{2}\right)\right)^{\frac{1}{2}}\right\|_{1}+\left\|\sum_{k=1}^{\infty}\left|d h_{k}\right|\right\|_{1}\right\} .
$$

Keeping the notation, our first weak type estimate reads as follows.

Theorem A. Let $f=\left(f_{1}, f_{2}, \ldots\right)$ be a bounded martingale in $L_{1}(\Omega)$. Then, we can decompose $f$ as a sum $f=g+h$ of two martingales adapted to the same filtration and satisfying the following inequality with an absolute constant $\mathrm{c}$

$\left(\mathrm{WB}_{1}\right)$

$$
\left\|\left(\sum_{k=1}^{\infty} \mathrm{E}_{k-1}\left(\left|d g_{k}\right|^{2}\right)\right)^{\frac{1}{2}}\right\|_{1, \infty}+\left\|\sum_{k=1}^{\infty}\left|d h_{k}\right|\right\|_{1, \infty} \leq \mathrm{c}\|f\|_{1} .
$$


The first relevant difficulty in proving Theorem A resides on the fact that we need to guess the right decomposition of $f$ before estimating the associated norms on the corresponding Hardy spaces. Note that this problem was avoided in [12] by using a duality argument, which is not available in our case. The solution to this problem turns out to be very elegant. Indeed, quite surprisingly the right decomposition is given by the classical Davis decomposition [5]. Moreover, the proof becomes quite involved since we combine Davis and Gundy decompositions to estimate the diagonal term. It is also worthy of mention that Theorem A provides an improvement of Davis decomposition. The reader is referred to the last section of this paper for the details.

The first term on the left of $\left(\mathrm{WB}_{1}\right)$ is clearly the natural weak analog of the first term on the right of $\left(B_{p}^{-}\right)$. However, the second term on the left of $\left(\mathrm{WB}_{1}\right)$ is only one possible interpretation for the corresponding $L_{p}$ term. Indeed, we have chosen the $L_{1, \infty}$ norm of the 1 -variation of $h$. In other words, the norm of the martingale difference sequence $d h$ in $L_{1, \infty}\left(\Omega ; \ell_{1}\right)$. This choice is motivated by the fact that the $L_{p}$ term is exactly the norm of $d h$ in $L_{p}\left(\Omega ; \ell_{p}\right)$. Another interpretation arises after rewriting $L_{p}\left(\Omega ; \ell_{p}\right)$ as the scalar-valued space $L_{p}\left(\Omega_{\oplus \infty}\right)$ where the associated measure is given by

$$
\mu_{\oplus \infty}\left(\bigoplus_{k \geq 1} \mathrm{~A}_{k}\right)=\sum_{k \geq 1} \mu\left(\mathrm{A}_{k}\right)
$$

In this case, the weak analog of the $p$-variation is given by

$$
\left\|\sum_{k=1}^{\infty} \delta_{k} \otimes d h_{k}\right\|_{L_{1, \infty}\left(\Omega_{\oplus \infty}\right)}=\sup _{\lambda>0} \lambda \sum_{k=1}^{\infty} \mu\left\{\left|d h_{k}\right|>\lambda\right\},
$$

where $\left(\delta_{k}\right)_{k \geq 1}$ denotes the canonical unit vector basis. At this point, it is worthy of mention that the norms of $L_{1, \infty}\left(\Omega ; \ell_{1}\right)$ and $L_{1, \infty}\left(\Omega_{\oplus \infty}\right)$ are not equivalent nor even comparable. Indeed, taking $\varphi_{k}=\chi_{[0,1 / k]}$ and $\xi_{k}=\frac{1}{k} \chi_{[0,1]}$, it is easy to check that

$$
\begin{aligned}
& \sup _{\lambda>0} \lambda \mu\left\{\sum_{k=1}^{m} \varphi_{k}>\lambda\right\} \sim 1<<\log m \sim \sup _{\lambda>0} \lambda \sum_{k=1}^{m} \mu\left\{\varphi_{k}>\lambda\right\}, \\
& \sup _{\lambda>0} \lambda \mu\left\{\sum_{k=1}^{m} \xi_{k}>\lambda\right\} \sim \log m>>1 \sim \sup _{\lambda>0} \lambda \sum_{k=1}^{m} \mu\left\{\xi_{k}>\lambda\right\} .
\end{aligned}
$$

Therefore, it makes sense to study the weak type estimate associated to this new interpretation of the $p$-variation. In order to state our second weak type estimate, we need to recall the notion of a regular filtration. The filtration 
$\mathrm{A}_{1}, \mathrm{~A}_{2}, \mathrm{~A}_{3}, \ldots$ of $\sigma$-subalgebras of $\mathrm{A}$ is called k-regular for some constant $\mathrm{k}>1$ if every non-negative martingale $f=\left(f_{1}, f_{2}, \ldots\right)$ adapted to this filtration satisfies

$$
f_{n} \leq \mathrm{k} f_{n-1} .
$$

Examples of regular martingales arise from the filtrations generated by bounded Vilenkin systems. In particular, the dyadic martingales are the most well-known examples of this kind, see [22] for more on this topic.

Theorem B. Let $f=\left(f_{1}, f_{2}, \ldots\right)$ be a bounded martingale in $L_{1}(\Omega)$ adapted to a k-regular filtration. Then, we can decompose $f$ as a sum $f=g+h$ of two martingales adapted to the same filtration and satisfying the following inequality with an absolute constant $\mathrm{c}$

$\left(\mathrm{WB}_{2}\right)\left\|\left(\sum_{k=1}^{\infty} \mathrm{E}_{k-1}\left(\left|d g_{k}\right|^{2}\right)\right)^{\frac{1}{2}}\right\|_{L_{1, \infty}(\Omega)}+\left\|\sum_{k=1}^{\infty} \delta_{k} \otimes d h_{k}\right\|_{L_{1, \infty}\left(\Omega_{\oplus \infty}\right)} \leq \mathrm{ck}\|f\|_{1}$.

The notion of k-regular filtration (equivalently that of previsible martingale) is necessary to formulate many martingale inequalities, see e.g. [1,2] or Chapter 2 in [22]. However, it is still unclear whether or not the k-regularity assumption in Theorem B is necessary. On one side, in view of some similar results in [2], it seems a natural condition. However, the proof we present here (see Paragraph 3.4 for a much simpler but less interesting one) gives some evidences that Theorem B might hold for general martingales with an absolute constant.

Nevertheless, even in the present form, Theorem B presents some advantages with respect to Theorem A. First, as we shall explain in the last section of this paper, it is much simpler to reprove Burkholder's inequality (via real interpolation and duality) starting from Theorem B. Second, our more elaborated proof of Theorem B goes further and gives rise to the result below.

Corollary C. Let $f=\left(f_{1}, f_{2}, \ldots\right)$ be a bounded martingale in $L_{1}(\Omega)$. Then, we can decompose each $f_{n}$ as a sum $f_{n}=g_{n}+h_{n}$ of two functions (nonnecessarily martingales) adapted to the same filtration and satisfying the following inequality with an absolute constant $\mathrm{c}$

$$
\left\|\left(\sum_{k=1}^{\infty} \mathrm{E}_{k-1}\left(\left|d g_{k}\right|^{2}\right)\right)^{\frac{1}{2}}\right\|_{L_{1, \infty}(\Omega)}+\left\|\sum_{k=1}^{\infty} \delta_{k} \otimes d h_{k}\right\|_{L_{1, \infty}\left(\Omega_{\oplus \infty}\right)} \leq \mathrm{c}\|f\|_{1} .
$$

At the time of this writing, Randrianantoanina independently obtained in [20] the noncommutative analogue of the result above. However, Theorems $\mathrm{A}$ and $\mathrm{B}$ have not been considered there. The noncommutative form of Corollary $\mathrm{C}$ has been applied to obtain the optimal constants in Junge/Xu's noncommutative Burkholder inequality. 
The reader familiar with the noncommutative setting will recognize the similarities between both papers. However, it is important to note that no background in noncommutative martingales is required to read this paper. In any case, we have decided to add a paragraph at the end explaining how both arguments are related (only for those readers familiar with the noncommutative theory). More concretely, although this is not mentioned in Randrianantoanina's paper, we shall explain how Davis decomposition appears (in a very indirect form) in [20].

Acknowledgements. I have received interesting suggestions and comments from Marius Junge, Teresa Martínez and Fernando Soria. I am specially indebted to Michael Cwikel for providing me an answer of Question 4.1 below. Finally, I would also like to thank the referee of this paper, whose suggestions led to an improved presentation.

\section{Martingale Decompositions}

Let us fix once and for all a probability space $(\Omega, A, \mu)$ and a filtration $\mathrm{A}_{1}, \mathrm{~A}_{2}, \ldots$ of $\sigma$-subalgebras of $\mathrm{A}$ with corresponding conditional expectations $E_{1}, E_{2}, \ldots$ Davis decomposition is a fundamental tool in the theory of martingale inequalities and it appeared for the first time in [5], where Davis applied it to prove his well-known theorem on the equivalence in $L_{1}(\Omega)$ between the martingale square function and Doob's maximal function

$$
\left\|f^{*}\right\|_{1} \sim_{\mathrm{c}}\left\|\left(\sum_{k=1}^{\infty}\left|d f_{k}\right|^{2}\right)^{\frac{1}{2}}\right\|_{1}
$$

Considering the truncated maximal functions

$$
f_{n}^{*}(w)=\sup _{1 \leq k \leq n}\left|f_{k}(w)\right|
$$

we formulate Davis decomposition $f=g+h$ by defining the differences

$$
\begin{aligned}
& d g_{k}=d f_{k} \chi_{\left\{f_{k}^{*}<2 f_{k-1}^{*}\right\}}-\mathrm{E}_{k-1}\left(d f_{k} \chi_{\left\{f_{k}^{*}<2 f_{k-1}^{*}\right\}}\right), \\
& d h_{k}=d f_{k} \chi_{\left\{f_{k}^{*} \geq 2 f_{k-1}^{*}\right\}}-\mathrm{E}_{k-1}\left(d f_{k} \chi_{\left\{f_{k}^{*} \geq 2 f_{k-1}^{*}\right\}}\right) .
\end{aligned}
$$

It is clear that $d g_{k}$ and $d h_{k}$ are martingale differences so that $g$ and $h$ become martingales adapted to the filtration $A_{1}, A_{2}, \ldots$ The properties stated in [5] and which appear in the literature are the following

$$
\left|d g_{k}\right| \leq 8 f_{k-1}^{*} \quad \text { and } \quad\left\|\sum_{k=1}^{\infty}\left|d h_{k}\right|\right\|_{p} \leq(4+4 p)\left\|f^{*}\right\|_{p}
$$


for $1 \leq p<\infty$, see e.g. [22] for an estimate of $g$ in the norm of the space of predictable martingales. The proof of these properties is rather simple, in contrast with their weak type analogs which arise from Theorem A, see Section 4.

Let us now describe Gundy's decomposition. Let $f=\left(f_{1}, f_{2}, \ldots\right)$ be a martingale on $(\Omega, \mathrm{A}, \mu)$ relative to the filtration fixed above that is bounded in $L_{1}(\Omega)$. Let $\lambda$ be a positive real number. Then we define the martingales $\alpha, \beta$ and $\gamma$ by their martingale differences

$$
\begin{aligned}
d \alpha_{k} & =d f_{k} \chi_{\left\{f_{k-1}^{*}>\lambda\right\}}, \\
d \beta_{k} & =d f_{k} \chi_{\left\{f_{k}^{*} \leq \lambda\right\}}-\mathrm{E}_{k-1}\left(d f_{k} \chi_{\left\{f_{k}^{*} \leq \lambda\right\}}\right), \\
d \gamma_{k} & =d f_{k} \chi_{\left\{f_{k-1}^{*} \leq \lambda<f_{k}^{*}\right\}}-\mathrm{E}_{k-1}\left(d f_{k} \chi_{\left\{f_{k-1}^{*} \leq \lambda<f_{k}^{*}\right\}}\right) .
\end{aligned}
$$

Again, these are clearly martingale differences with sum $d f_{k}$ and thus we get a decomposition $f=\alpha+\beta+\gamma$ into three martingales. This decomposition is in fact due to Burkholder [1] and is simpler than the one originally formulated by Gundy [7]. Indeed, the decomposition stated above uses only one stopping time while the one originally formulated by Gundy needs two stopping times. I learned this simpler (but weaker, see below) decomposition from Narcisse Randrianantoanina. The following are the properties satisfied by the given decomposition.

i) The martingale $\alpha$ satisfies

$$
\lambda \mu\left\{\sum_{k=1}^{\infty}\left|d \alpha_{k}\right|>0\right\} \leq \mathrm{c}\|f\|_{1} .
$$

ii) The martingale $\beta$ satisfies

$$
\|\beta\|_{1} \leq \mathrm{c}\|f\|_{1} \text { and } \frac{1}{\lambda}\|\beta\|_{2}^{2} \leq \mathrm{c}\|f\|_{1} .
$$

iii) The martingale $\gamma$ satisfies

$$
\sum_{k=1}^{\infty}\left\|d \gamma_{k}\right\|_{1} \leq \mathrm{c}\|f\|_{1} .
$$

Gundy's original decomposition, paying the price of using two stopping times, obtains the additional estimate $\|\beta\|_{\infty} \leq \mathrm{c} \lambda$. In particular, we can control any $L_{p}$ norm of $\beta$ by means of Hölder's inequality. Nevertheless, we shall only need the $L_{2}$ estimate in this paper. Gundy's decomposition theorem plays a central role in classical martingale theory and it can be regarded as a probabilistic counterpart of the well-known Calderón-Zygmund decomposition for integrable functions in harmonic analysis, see $[6,7]$ for further details. 


\section{Proof of Theorem A}

We first observe that we may assume without lost of generality that $f$ is a positive martingale. Indeed, otherwise we can always decompose $f_{n}$ into a linear combination of four positive functions

$$
f_{n}=\left(f_{n}^{(1)}-f_{n}^{(2)}\right)+i\left(f_{n}^{(3)}-f_{n}^{(4)}\right) .
$$

According to a classical result due to Krickeberg, it turns out that this provides a martingale decomposition of $f$ into four positive martingales. Therefore, since we have the inequality

$$
\sum_{k=1}^{4}\left\|f_{n}^{(k)}\right\|_{1} \leq 2\left\|f_{n}\right\|_{1}
$$

and the expressions on the left of $\left(\mathrm{WB}_{1}\right)$ clearly satisfy a quasi-triangle inequality, we may assume that $f$ is positive. On the other hand, as we have anticipated in the Introduction, the right decomposition to prove the weak Burkholder inequality is Davis decomposition

$$
\begin{aligned}
& d g_{k}=d f_{k} \chi_{\left\{f_{k}^{*}<2 f_{k-1}^{*}\right\}}-\mathrm{E}_{k-1}\left(d f_{k} \chi_{\left\{f_{k}^{*}<2 f_{k-1}^{*}\right\}}\right), \\
& d h_{k}=d f_{k} \chi_{\left\{f_{k}^{*} \geq 2 f_{k-1}^{*}\right\}}-\mathrm{E}_{k-1}\left(d f_{k} \chi_{\left\{f_{k}^{*} \geq 2 f_{k-1}^{*}\right\}}\right) .
\end{aligned}
$$

In what follows, c might have different values from one instance to another.

\subsection{Step 1: Proof of the estimate}

$$
\left\|\left(\sum_{k=1}^{\infty} \mathrm{E}_{k-1}\left(\left|d g_{k}\right|^{2}\right)\right)^{\frac{1}{2}}\right\|_{1, \infty} \leq \mathrm{c}\|f\|_{1} .
$$

Taking $\tau_{k}=d f_{k} \chi_{\left\{f_{k}^{*}<2 f_{k-1}^{*}\right\}}$, we have

$$
\mathrm{E}_{k-1}\left(\left|d g_{k}\right|^{2}\right)=\mathrm{E}_{k-1}\left(\left|\tau_{k}\right|^{2}+\left|\mathrm{E}_{k-1}\left(\tau_{k}\right)\right|^{2}-2 \tau_{k} \mathrm{E}_{k-1}\left(\tau_{k}\right)\right) \leq \mathrm{E}_{k-1}\left(\left|\tau_{k}\right|^{2}\right) .
$$

Thus, we may replace $d g_{k}$ by $\tau_{k}$ and defining the function

$$
\Phi=\sum_{k=1}^{\infty} \mathrm{E}_{k-1}\left(\left|d f_{k}\right|^{2} \chi_{\left\{f_{k}^{*}<2 f_{k-1}^{*}\right\}}\right),
$$

it suffices to prove that $\lambda \mu\left(\Phi>\lambda^{2}\right) \leq \mathrm{c}\|f\|_{1}$ for all $\lambda>0$. For fixed $\lambda$ we have

$$
\lambda \mu\left(\Phi>\lambda^{2}\right) \leq \lambda \mu\left(f^{*}>\lambda\right)+\lambda \mu\left(\chi_{\left\{f^{*} \leq \lambda\right\}} \Phi>\lambda^{2} / 2\right) .
$$


Therefore, since $\mathrm{E}_{0}=\mathrm{E}_{1}$ and $\chi_{\left\{f^{*} \leq \lambda\right\}} \leq \chi_{\left\{f_{k-1}^{*} \leq \lambda\right\}}$ for $k \geq 2$, we have

$$
\lambda \mu\left(\Phi>\lambda^{2}\right) \leq \lambda \mu\left(f^{*}>\lambda\right)+\lambda \mu\left(\sum_{k=1}^{\infty} \mathrm{E}_{k-1}\left(\left|d f_{k}\right|^{2} \chi_{\left\{f_{k}^{*} \leq 2 \lambda\right\}}\right)>\lambda^{2} / 2\right) .
$$

According to Doob's maximal inequality, the first term is controlled by $\mathrm{c}\|f\|_{1}$. On the other hand, in order to estimate the second term, we need to decompose it into two pieces. We use Chebychev's inequality

$$
\begin{aligned}
\lambda \mu\left(\sum_{k=1}^{\infty} \mathrm{E}_{k-1}\left(\left|d f_{k}\right|^{2} \chi_{\left\{f_{k}^{*} \leq 2 \lambda\right\}}\right)>\lambda^{2} / 2\right) & \leq \frac{2}{\lambda}\left\|\sum_{k=1}^{\infty} \mathrm{E}_{k-1}\left(\left|d f_{k}\right|^{2} \chi_{\left\{f_{k}^{*} \leq 2 \lambda\right\}}\right)\right\|_{1}=\frac{2}{\lambda} \sum_{k=1}^{\infty}\left\|d f_{k} \chi_{\left\{f_{k}^{*} \leq 2 \lambda\right\}}\right\|_{2}^{2} \\
\leq & \frac{4}{\lambda} \sum_{k=1}^{\infty}\left\|f_{k} \chi_{\left\{f_{k}^{*} \leq 2 \lambda\right\}}-f_{k-1} \chi_{\left\{f_{k-1}^{*} \leq 2 \lambda\right\}}\right\|_{2}^{2} \\
& +\frac{4}{\lambda} \sum_{k=2}^{\infty}\left\|f_{k-1} \chi_{\left\{f_{k-1}^{*} \leq 2 \lambda\right\}}-f_{k-1} \chi_{\left\{f_{k}^{*} \leq 2 \lambda\right\}}\right\|_{2}^{2} .
\end{aligned}
$$

Denoting by $\mathrm{A}$ and $\mathrm{B}$ the two terms on the right, we have

$$
\begin{aligned}
\mathrm{A}= & \frac{4}{\lambda} \sum_{k=1}^{\infty} \int_{\Omega} f_{k}^{2} \chi_{\left\{f_{k}^{*} \leq 2 \lambda\right\}}-f_{k-1}^{2} \chi_{\left\{f_{k-1}^{*} \leq 2 \lambda\right\}} d \mu \\
& +\frac{8}{\lambda} \sum_{k=2}^{\infty} \int_{\Omega} f_{k-1} \chi_{\left\{f_{k-1}^{*} \leq 2 \lambda\right\}}\left(f_{k-1}-f_{k} \chi_{\left\{f_{k}^{*} \leq 2 \lambda\right\}}\right) d \mu \\
= & \frac{4}{\lambda} \lim _{n \rightarrow \infty} \int_{\Omega} f_{n}^{2} \chi_{\left\{f_{n}^{*} \leq 2 \lambda\right\}} d \mu \\
& +\frac{8}{\lambda} \sum_{k=2}^{\infty} \int_{\Omega} f_{k-1} \chi_{\left\{f_{k-1}^{*} \leq 2 \lambda\right\}}\left(f_{k-1} \chi_{\left\{f_{k-1}^{*} \leq 2 \lambda\right\}}-\mathrm{E}_{k-1}\left(f_{k} \chi_{\left\{f_{k}^{*} \leq 2 \lambda\right\}}\right)\right) d \mu \\
\leq & \frac{4}{\lambda} \lim _{n \rightarrow \infty}\left\|f_{n}\right\|_{1}\left\|f_{n} \chi_{\left\{f_{n}^{*} \leq 2 \lambda\right\}}\right\|_{\infty} \\
& +\frac{8}{\lambda} \sum_{k=2}^{\infty}\left\|f_{k-1} \chi_{\left\{f_{k-1}^{*} \leq 2 \lambda\right\}}\right\|_{\infty} \int_{\Omega}\left|f_{k-1} \chi_{\left\{f_{k-1}^{*} \leq 2 \lambda\right\}}-\mathrm{E}_{k-1}\left(f_{k} \chi_{\left\{f_{k}^{*} \leq 2 \lambda\right\}}\right)\right| d \mu .
\end{aligned}
$$

This gives

$$
\mathrm{A} \leq 8\|f\|_{1}+16 \sum_{k=2}^{\infty} \int_{\Omega}\left|f_{k-1} \chi_{\left\{f_{k-1}^{*} \leq 2 \lambda\right\}}-\mathrm{E}_{k-1}\left(f_{k} \chi_{\left\{f_{k}^{*} \leq 2 \lambda\right\}}\right)\right| d \mu .
$$

However, we may drop the modulus in the integral since

$$
\mathrm{E}_{k-1}\left(f_{k} \chi_{\left\{f_{k}^{*} \leq 2 \lambda\right\}}\right) \leq \mathrm{E}_{k-1}\left(f_{k} \chi_{\left\{f_{k-1}^{*} \leq 2 \lambda\right\}}\right)=f_{k-1} \chi_{\left\{f_{k-1}^{*} \leq 2 \lambda\right\}} .
$$


In that case, $\mathrm{E}_{k-1}$ disappears and we obtain a telescopic sum of decreasing terms

$$
\mathrm{A} \leq 8\|f\|_{1}+16 \sum_{k=2}^{\infty} \int_{\Omega} f_{k-1} \chi_{\left\{f_{k-1}^{*} \leq 2 \lambda\right\}} d \mu-\int_{\Omega} f_{k} \chi_{\left\{f_{k}^{*} \leq 2 \lambda\right\}} d \mu \leq 24\|f\|_{1} .
$$

It remains to estimate the term B. This term is much easier to handle

$$
\begin{aligned}
\mathrm{B} & =\frac{4}{\lambda} \sum_{k=2}^{\infty}\left\|f_{k-1} \chi_{\left\{f_{k-1}^{*} \leq 2 \lambda<f_{k}^{*}\right\}}\right\|_{2}^{2} \\
& \leq \frac{4}{\lambda} \sum_{k=2}^{\infty}\left\|\chi_{\left\{f_{k-1}^{*} \leq 2 \lambda<f_{k}^{*}\right\}}\right\|_{1}\left\|f_{k-1}^{2} \chi_{\left\{f_{k-1}^{*} \leq 2 \lambda<f_{k}^{*}\right\}}\right\|_{\infty} \\
& \leq 16 \lambda \sum_{k=2}^{\infty} \int_{\Omega} \chi_{\left\{f_{k-1}^{*} \leq 2 \lambda\right\}}-\chi_{\left\{f_{k}^{*} \leq 2 \lambda\right\}} d \mu \leq 16 \lambda \mu\left(f^{*}>2 \lambda\right) \leq 8\|f\|_{1} .
\end{aligned}
$$

\subsection{Step 2: Proof of the estimate}

$$
\left\|\sum_{k=1}^{\infty}\left|d h_{k}\right|\right\|_{1, \infty} \leq \mathrm{c}\|f\|_{1}
$$

Taking into account the form of $d h_{k}$ according to Davis decomposition, we have

$$
\begin{aligned}
\left\|\sum_{k=1}^{\infty}\left|d h_{k}\right|\right\|_{1, \infty} \leq & 2\left\|\sum_{k=1}^{\infty}\left|d f_{k} \chi_{\left\{f_{k}^{*} \geq 2 f_{k-1}^{*}\right\}}\right|\right\|_{1, \infty} \\
& +2\left\|\sum_{k=1}^{\infty}\left|\mathrm{E}_{k-1}\left(d f_{k} \chi_{\left\{f_{k}^{*} \geq 2 f_{k-1}^{*}\right\}}\right)\right|\right\|_{1, \infty}=\mathrm{C}+\mathrm{D} .
\end{aligned}
$$

The estimate for the term $\mathrm{C}$ is very simple. Indeed, it suffices to use the classical property of this part of Davis decomposition. Namely, we have $f_{k}^{*} \geq 2 f_{k-1}^{*}$ if and only if $f_{k}^{*} \leq 2\left(f_{k}^{*}-f_{k-1}^{*}\right)$. In particular, we deduce

$$
\left|d f_{k} \chi_{\left\{f_{k}^{*} \geq 2 f_{k-1}^{*}\right\}}\right| \leq 2 f_{k}^{*} \chi_{\left\{f_{k}^{*} \leq 2\left(f_{k}^{*}-f_{k-1}^{*}\right)\right\}} \leq 4\left(f_{k}^{*}-f_{k-1}^{*}\right)
$$

and conclude the following estimate

$$
\mathrm{C} \leq 8 \sup _{\lambda>0} \lambda \mu\left\{\sum_{k=1}^{\infty} f_{k}^{*}-f_{k-1}^{*}>\lambda\right\}=8 \sup _{\lambda>0} \lambda \mu\left(f^{*}>\lambda\right) \leq 8\|f\|_{1} .
$$

The estimate for $\mathrm{D}$ is a little more complicated, we have

$$
\mathrm{D}=2 \sup _{\lambda>0} \lambda \mu\left\{\sum_{k=1}^{\infty}\left|\mathrm{E}_{k-1}\left(d f_{k} \chi_{\left\{f_{k}^{*} \geq 2 f_{k-1}^{*}\right\}}\right)\right|>\lambda\right\} .
$$


Then we fix $\lambda>0$ and apply Gundy's decomposition (2) to $f$

$$
d f_{k}=d \alpha_{k}+d \beta_{k}+d \gamma_{k}
$$

By the quasi-triangle inequality we may write

$$
\begin{aligned}
& \lambda \mu\left\{\sum_{k=1}^{\infty}\left|\mathrm{E}_{k-1}\left(d f_{k} \chi_{\left\{f_{k}^{*} \geq 2 f_{k-1}^{*}\right\}}\right)\right|>\lambda\right\} \\
& \leq \lambda \mu\left\{\sum_{k=1}^{\infty} \mathrm{E}_{k-1}\left(\left|d \alpha_{k}\right| \chi_{\left\{f_{k}^{*} \geq 2 f_{k-1}^{*}\right\}}\right)>\frac{\lambda}{3}\right\} \\
&+\lambda \mu\left\{\sum_{k=1}^{\infty} \mathrm{E}_{k-1}\left(\left|d \beta_{k}\right| \chi_{\left\{f_{k}^{*} \geq 2 f_{k-1}^{*}\right\}}\right)>\frac{\lambda}{3}\right\} \\
&+\lambda \mu\left\{\sum_{k=1}^{\infty} \mathrm{E}_{k-1}\left(\left|d \gamma_{k}\right| \chi_{\left\{f_{k}^{*} \geq 2 f_{k-1}^{*}\right\}}\right)>\frac{\lambda}{3}\right\} .
\end{aligned}
$$

We shall denote these terms by $\mathrm{D}_{\alpha}, \mathrm{D}_{\beta}$ and $\mathrm{D}_{\gamma}$ respectively. The estimates for $\alpha$ and $\gamma$ are straightforward. Indeed, recalling that $d \alpha_{k}=d f_{k} \chi_{\left\{f_{k-1}^{*}>\lambda\right\}}$ and that the function $\chi_{\left\{f_{k-1}^{*}>\lambda\right\}}$ is predictable, we conclude

$\mathrm{D}_{\alpha}=\lambda \mu\left\{\sum_{k=1}^{\infty} \chi_{\left\{f_{k-1}^{*}>\lambda\right\}} \mathrm{E}_{k-1}\left(\left|d f_{k}\right| \chi_{\left\{f_{k}^{*} \geq 2 f_{k-1}^{*}\right\}}\right)>\frac{\lambda}{3}\right\} \leq \lambda \mu\left(f^{*}>\lambda\right) \leq\|f\|_{1}$.

On the other hand, by Chebychev's inequality we deduce

$$
\mathrm{D}_{\gamma} \leq 3\left\|\sum_{k=1}^{\infty} \mathrm{E}_{k-1}\left(\left|d \gamma_{k}\right| \chi_{\left\{f_{k}^{*} \geq 2 f_{k-1}^{*}\right\}}\right)\right\|_{1} \leq 3 \sum_{k=1}^{\infty}\left\|d \gamma_{k}\right\|_{1} \leq \mathrm{c}\|f\|_{1},
$$

where the last inequality follows from property iii) in Gundy's decomposition, see Section 1. It only remains to estimate the term $\mathrm{D}_{\beta}$. To that aim, we first recall the dual form of Doob's maximal inequality, since we shall need it in the proof. Given $1 \leq p<\infty$ and a sequence $\left(\psi_{m}\right)_{m \geq 1}$ of positive functions in $L_{p}(\Omega)$, we have

$$
\left\|\sum_{m} \mathrm{E}_{m-1}\left(\phi_{m}\right)\right\|_{p} \leq \mathrm{cp}\left\|\sum_{m} \phi_{m}\right\|_{p}
$$

I learned this from Marius Junge, see Burkholder's paper [1] for more details. We now apply Chebychev's inequality for $p=3$ and the dual Doob 
inequality (3) for the same index, we obtain

$$
\begin{aligned}
\mathrm{D}_{\beta} & \leq \mathrm{c} \lambda^{-2}\left\|\sum_{k=1}^{\infty} \mathrm{E}_{k-1}\left(\left|d \beta_{k}\right| \chi_{\left\{f_{k}^{*} \geq 2 f_{k-1}^{*}\right\}}\right)\right\|_{3}^{3} \\
& \leq \mathrm{c} \lambda^{-2}\left\|\sum_{k=1}^{\infty}\left|d \beta_{k}\right| \chi_{\left\{f_{k}^{*} \geq 2 f_{k-1}^{*}\right\}}\right\|_{3}^{3} \\
& \leq \mathrm{c} \lambda^{-2}\left\|\left(\sum_{k=1}^{\infty}\left|d \beta_{k}\right|^{2}\right)^{\frac{1}{3}}\left(\sum_{k=1}^{\infty}\left|d \beta_{k}\right|^{\frac{1}{2}} \chi_{\left\{f_{k}^{*} \geq 2 f_{k-1}^{*}\right\}}\right)^{\frac{2}{3}}\right\|_{3}^{3} \\
& =\mathrm{c} \lambda^{-2}\left\|\left(\sum_{k=1}^{\infty}\left|d \beta_{k}\right|^{2}\right)\left(\sum_{k=1}^{\infty}\left|d \beta_{k}\right|^{\frac{1}{2}} \chi_{\left\{f_{k}^{*} \geq 2 f_{k-1}^{*}\right\}}\right)^{2}\right\|_{1} \\
& \leq \mathrm{c} \lambda^{-2}\left\|\sum_{k=1}^{\infty}\left|d \beta_{k}\right|^{2}\right\|\left\|_{1}\right\| \sum_{k=1}^{\infty}\left|d \beta_{k}\right|^{\frac{1}{2}} \chi_{\left\{f_{k}^{*} \geq 2 f_{k-1}^{*}\right\}} \|_{\infty}^{2} .
\end{aligned}
$$

Thus, by the quadratic estimate in Gundy's decomposition (see Section 1)

$$
\mathrm{D}_{\beta} \leq \mathrm{c} \lambda^{-1}\left\|\sum_{k=1}^{\infty}\left|d \beta_{k}\right|^{\frac{1}{2}} \chi_{\left\{f_{k}^{*} \geq 2 f_{k-1}^{*}\right\}}\right\|_{\infty}^{2}\|f\|_{1} .
$$

Therefore, the only remaining estimate to conclude the proof of Theorem A is the following

$$
\left\|\sum_{k=1}^{\infty}\left|d \beta_{k}\right|^{\frac{1}{2}} \chi_{\left\{f_{k}^{*} \geq 2 f_{k-1}^{*}\right\}}\right\|_{\infty} \leq \mathrm{c} \sqrt{\lambda} .
$$

In order to prove inequality (4) we write $d \beta_{k}$ as follows

$$
\begin{aligned}
d \beta_{k}= & f_{k} \chi_{\left\{f_{k}^{*} \leq \lambda\right\}}-f_{k-1} \chi_{\left\{f_{k}^{*} \leq \lambda\right\}} \\
& +\mathrm{E}_{k-1}\left(f_{k-1} \chi_{\left\{f_{k}^{*} \leq \lambda\right\}}\right)-\mathrm{E}_{k-1}\left(f_{k} \chi_{\left\{f_{k}^{*} \leq \lambda\right\}}\right)=a_{k}-b_{k}+c_{k}-d_{k} .
\end{aligned}
$$

We clearly have

$$
\max \left(a_{k}, b_{k}\right) \leq f_{k}^{*} \chi_{\left\{f_{k}^{*} \leq \lambda\right\}}
$$

On the other hand, the following estimates hold

$$
\begin{aligned}
& c_{k}=f_{k-1} \mathrm{E}_{k-1}\left(\chi_{\left\{f_{k}^{*} \leq \lambda\right\}}\right) \leq f_{k-1} \mathrm{E}_{k-1}\left(\chi_{\left\{f_{k-1}^{*} \leq \lambda\right\}}\right) \leq f_{k-1}^{*} \chi_{\left\{f_{k-1}^{*} \leq \lambda\right\}}, \\
& d_{k} \leq \mathrm{E}_{k-1}\left(f_{k} \chi_{\left\{f_{k-1}^{*} \leq \lambda\right\}}\right)=f_{k-1} \chi_{\left\{f_{k-1}^{*} \leq \lambda\right\}} \leq f_{k-1}^{*} \chi_{\left\{f_{k-1}^{*} \leq \lambda\right\}} .
\end{aligned}
$$

Thus we conclude

$$
\left|d \beta_{k}\right|^{\frac{1}{2}} \leq \sqrt{a_{k}+b_{k}}+\sqrt{c_{k}+d_{k}} \leq \sqrt{2}\left(\sqrt{f_{k}^{*}} \chi_{\left\{f_{k}^{*} \leq \lambda\right\}}+\sqrt{f_{k-1}^{*}} \chi_{\left\{f_{k-1}^{*} \leq \lambda\right\}}\right) .
$$


This means that it suffices to prove the following estimates

$$
\begin{aligned}
& \mathrm{E}=\left\|\sum_{k=1}^{\infty} \sqrt{f_{k}^{*}} \chi_{\left\{f_{k}^{*} \leq \lambda\right\}} \chi_{\left\{f_{k}^{*} \geq 2 f_{k-1}^{*}\right\}}\right\|_{\infty} \leq \mathrm{c} \sqrt{\lambda}, \\
& \mathrm{F}=\left\|\sum_{k=2}^{\infty} \sqrt{f_{k-1}^{*}} \chi_{\left\{f_{k-1}^{*} \leq \lambda\right\}} \chi_{\left\{f_{k}^{*} \geq 2 f_{k-1}^{*}\right\}}\right\|_{\infty} \leq \mathrm{c} \sqrt{\lambda} .
\end{aligned}
$$

\subsection{Step 3: Proof of the estimate}

$$
\max (\mathrm{E}, \mathrm{F}) \leq \frac{\sqrt{2}}{\sqrt{2}-1} \sqrt{\lambda}
$$

Fix $w \in \Omega$ and set

$$
\mathcal{S}_{\mathrm{E}, n}(w)=\sum_{k=1}^{n} \phi_{k}(w)=\sum_{k=1}^{n} \sqrt{f_{k}^{*}(w)} \chi_{\left\{f_{k}^{*} \leq \lambda\right\}}(w) \chi_{\left\{f_{k}^{*} \geq 2 f_{k-1}^{*}\right\}}(w) .
$$

We shall prove by induction on $n$ that $\mathcal{S}_{\mathrm{E}, n}(w) \leq \sqrt{2 \lambda} /(\sqrt{2}-1)$. Indeed, the assertion is clear for $n=1$. Thus, let us assume that the assertion holds for $n-1$ and let us estimate $\mathcal{S}_{\mathrm{E}, n}(w)$. If $\phi_{n}(w)=0$ we have $\mathcal{S}_{\mathrm{E}, n}(w)=\mathcal{S}_{\mathrm{E}, n-1}(w)$ and there is nothing to prove. If $\phi_{n}(w) \neq 0$, we must have $f_{n}^{*}(w) \leq \lambda$ and

$$
f_{n-1}^{*}(w) \leq \frac{1}{2} f_{n}^{*}(w) \leq \lambda / 2 .
$$

Then, if $\phi_{n-1}(w) \neq 0$ we know that

$$
f_{n-1}^{*}(w) \leq \lambda / 2 \quad \text { and } \quad f_{n-2}^{*}(w) \leq \frac{1}{2} f_{n-1}^{*}(w) \leq \lambda / 4 .
$$

On the other hand, if $\phi_{n-1}(w)=0$ we may ignore that term in the sum and we still have at our disposal that $f_{n-2}^{*}(w) \leq f_{n-1}^{*}(w) \leq \lambda / 2$, so that we can argue in the same way for $\phi_{n-2}(w)$. Iterating the same argument, it is not difficult to conclude that

$$
\mathcal{S}_{\mathrm{E}, n}(w) \leq \sqrt{\lambda} \sum_{k=0}^{\infty}\left(\frac{1}{\sqrt{2}}\right)^{k}=\frac{\sqrt{2}}{\sqrt{2}-1} \sqrt{\lambda} .
$$

Since this argument works for any $w \in \Omega$, we have proved our claim for E. Arguing in a similar way, we obtain the same bound for F. Let us include the details for the sake of completeness. Fix $w \in \Omega$ and set

$$
\mathcal{S}_{\mathrm{F}, n}(w)=\sum_{k=2}^{n} \psi_{k}(w)=\sum_{k=2}^{n} \sqrt{f_{k-1}^{*}(w)} \chi_{\left\{f_{k-1}^{*} \leq \lambda\right\}}(w) \chi_{\left\{f_{k}^{*} \geq 2 f_{k-1}^{*}\right\}}(w) .
$$


Again, it is clear that $\psi_{2}(w) \leq \sqrt{\lambda}$ so that we may proceed by induction on $n$ and assume that the inequality $\mathcal{S}_{\mathrm{F}, n-1}(w) \leq \sqrt{2 \lambda} /(\sqrt{2}-1)$ holds. If $\psi_{n}(w)=0$ there is nothing to prove while for $\psi_{n}(w) \neq 0$ we deduce that $f_{n-1}^{*}(w) \leq \lambda$. Going backwards, we seek for the next non-zero term $\psi_{j}(w) \neq 0$. Such term implies

$$
f_{j-1}^{*}(w) \leq \frac{1}{2} f_{j}^{*}(w) \leq \frac{1}{2} f_{n-1}^{*}(w) \leq \lambda / 2 .
$$

Iterating one more time we conclude

$$
\mathcal{S}_{\mathrm{F}, n}(w) \leq \sqrt{\lambda} \sum_{k=0}^{\infty}\left(\frac{1}{\sqrt{2}}\right)^{k}=\frac{\sqrt{2}}{\sqrt{2}-1} \sqrt{\lambda}
$$

This justifies our claim

$$
\max (\mathrm{E}, \mathrm{F}) \leq \frac{\sqrt{2}}{\sqrt{2}-1} \sqrt{\lambda}
$$

which implies (5) and (6). Thus, the proof of Theorem A is completed.

Remark 2.1. As it was observed by the referee, the martingale decomposition implicit in Theorem A can be adapted to the case of continuous martingales, at least for the case $1<p<2$. More precisely, if $f=(f(\xi))_{\xi \in \Lambda}$ is a continuous martingale bounded in $L_{p}(\Omega)$, we may find a martingale decomposition $f=g+h$ such that

$$
\begin{array}{r}
\left\|\left(\sum_{k=1}^{n} \mathrm{E}_{\xi_{k-1}}\left(\left|g\left(\xi_{k}\right)-g\left(\xi_{k-1}\right)\right|^{2}\right)\right)^{\frac{1}{2}}\right\|_{p}+\left\|\sum_{k=1}^{n}\left|h\left(\xi_{k}\right)-h\left(\xi_{k-1}\right)\right|\right\|_{p} \\
\leq \mathrm{c}_{p}\|f\|_{p}
\end{array}
$$

holds for every partition $\pi=\left\{\xi_{0}<\xi_{1}<\ldots<\xi_{n}\right\} \subset \Lambda$. Indeed, if $f_{\pi}$ denotes the restriction of $f$ to $\pi$, inequality (7) holds for $f_{\pi}=g_{\pi}+h_{\pi}$ by Theorem A and the real interpolation method. The desired decomposition then arises from a suitable weak* limit. More precisely, note that both terms in (7) are self-improving in the following sense, if $\pi$ is finer than $\pi^{\prime}$ then $\left(g_{\pi}, h_{\pi}\right)$ satisfy $(7)$ for $\pi^{\prime}$. Therefore, we may obtain $g$ (resp. $h$ ) as a cluster point of the family of $g_{\pi}$ 's (resp. $h_{\pi}$ 's) in the weak* topology. Recall that the conditional Hardy space is reflexive (this is even true in the noncommutative setting, see [12] for details) and $L_{p}\left(\Omega, \ell_{1}\right)$ is a dual Banach space. At the time of this writing we do not know whether this inequality can be generalized to the weak $L_{1, \infty}$ situation. 


\section{Proof of Theorem B}

Our aim in this section is proving Theorem B. However, instead of giving the simplest proof available (see Paragraph 3.4 for this), we present a more general proof where the hypothesis of k-regularity is only needed in the very last step. This somehow supports our comment in the Introduction on the validity of Theorem B for non-regular martingales. Moreover, as we shall see below, our proof goes a little further, see Corollary C below.

For now on and until the very end of the proof, we assume that $f$ is a bounded martingale in $L_{1}(\Omega)$ adapted to a non-necessarily regular filtration. Our martingale decomposition $f=g+h$ is given one more time by Davis decomposition

$$
\begin{aligned}
& d g_{k}=d f_{k} \chi_{\left\{f_{k}^{*}<2 f_{k-1}^{*}\right\}}-\mathrm{E}_{k-1}\left(d f_{k} \chi_{\left\{f_{k}^{*}<2 f_{k-1}^{*}\right\}}\right), \\
& d h_{k}=d f_{k} \chi_{\left\{f_{k}^{*} \geq 2 f_{k-1}^{*}\right\}}-\mathrm{E}_{k-1}\left(d f_{k} \chi_{\left\{f_{k}^{*} \geq 2 f_{k-1}^{*}\right\}}\right) .
\end{aligned}
$$

In particular, the weak type estimate

$$
\left\|\left(\sum_{k=1}^{\infty} \mathrm{E}_{k-1}\left(\left|d g_{k}\right|^{2}\right)\right)^{\frac{1}{2}}\right\|_{L_{1, \infty}(\Omega)} \leq \mathrm{c}\|f\|_{1}
$$

holds with an absolute constant $\mathrm{c}$ by means of Theorem A.

In order to estimate the second term on the left of $\left(\mathrm{WB}_{2}\right)$ we combine one more time Davis and Gundy decompositions. More concretely, for fixed $\lambda>0$ and according to (2) we have

$$
\begin{aligned}
d h_{k}= & d \alpha_{k} \chi_{\left\{f_{k}^{*} \geq 2 f_{k-1}^{*}\right\}}-\mathrm{E}_{k-1}\left(d \alpha_{k} \chi_{\left\{f_{k}^{*} \geq 2 f_{k-1}^{*}\right\}}\right) \\
& +d \beta_{k} \chi_{\left\{f_{k}^{*} \geq 2 f_{k-1}^{*}\right\}}-\mathrm{E}_{k-1}\left(d \beta_{k} \chi_{\left\{f_{k}^{*} \geq 2 f_{k-1}^{*}\right\}}\right) \\
& +d \gamma_{k} \chi_{\left\{f_{k}^{*} \geq 2 f_{k-1}^{*}\right\}}-\mathrm{E}_{k-1}\left(d \gamma_{k} \chi_{\left\{f_{k}^{*} \geq 2 f_{k-1}^{*}\right\}}\right) \\
= & d h_{\alpha k}+d h_{\beta k}+d h_{\gamma k} .
\end{aligned}
$$

As in Theorem A, the terms associated to $\gamma$ are the simplest ones

$$
\begin{aligned}
\left\|\sum_{k=1}^{\infty} \delta_{k} \otimes d \gamma_{k} \chi_{\left\{f_{k}^{*} \geq 2 f_{k-1}^{*}\right\}}\right\|_{L_{1, \infty}\left(\Omega_{\oplus \infty}\right)} & \leq \sum_{k=1}^{\infty}\left\|d \gamma_{k}\right\|_{1} \leq \mathrm{c}\|f\|_{1}, \\
\left\|\sum_{k=1}^{\infty} \delta_{k} \otimes \mathrm{E}_{k-1}\left(d \gamma_{k} \chi_{\left\{f_{k}^{*} \geq 2 f_{k-1}^{*}\right\}}\right)\right\|_{L_{1, \infty}\left(\Omega_{\oplus \infty}\right)} & \leq \sum_{k=1}^{\infty}\left\|d \gamma_{k}\right\|_{1} \leq \mathrm{c}\|f\|_{1} .
\end{aligned}
$$

Therefore, it remains to estimate the terms associated to $\alpha$ and $\beta$. 


\subsection{Step 1: Proof of the estimate}

$$
\lambda \sum_{k=1}^{\infty} \mu\left\{\left|d h_{\beta k}\right|>\lambda\right\} \leq \mathrm{c}\|f\|_{1} .
$$

This estimate is similar to that of Theorem A. By Chebychev's inequality

$$
\begin{aligned}
\lambda \sum_{k=1}^{\infty} \mu\left\{\left|d \beta_{k}\right| \chi_{\left\{f_{k}^{*} \geq 2 f_{k-1}^{*}\right\}}>\lambda\right\} & \leq \lambda^{-2} \sum_{k=1}^{\infty}\left\|\left|d \beta_{k}\right| \chi_{\left\{f_{k}^{*} \geq 2 f_{k-1}^{*}\right\}}\right\|_{3}^{3} \\
& =\lambda^{-2}\left\|\sum_{k=1}^{\infty}\left|d \beta_{k}\right|^{3} \chi_{\left\{f_{k}^{*} \geq 2 f_{k-1}^{*}\right\}}\right\|_{1} \\
& \leq \lambda^{-2}\left\|\left(\sum_{k=1}^{\infty}\left|d \beta_{k}\right|^{2}\right) \sup _{k \geq 1}\left|d \beta_{k}\right| \chi_{\left\{f_{k}^{*} \geq 2 f_{k-1}^{*}\right\}}\right\|_{1}
\end{aligned}
$$

Now we observe that $\left|d \beta_{k}\right| \leq 4 \lambda$ for all integer $k \geq 1$, since

$$
\begin{aligned}
\left|d \beta_{k}\right| & =\left|d f_{k} \chi_{\left\{f_{k}^{*} \leq \lambda\right\}}-\mathrm{E}_{k-1}\left(d f_{k} \chi_{\left\{f_{k}^{*} \leq \lambda\right\}}\right)\right| \\
& \leq 2 f_{k}^{*} \chi_{\left\{f_{k}^{*} \leq \lambda\right\}}+2 \mathrm{E}_{k-1}\left(f_{k}^{*} \chi_{\left\{f_{k}^{*} \leq \lambda\right\}}\right) \leq 4 \lambda .
\end{aligned}
$$

Therefore, using the quadratic estimate in Gundy's decomposition

$$
\lambda \sum_{k=1}^{\infty} \mu\left\{\left|d \beta_{k}\right| \chi_{\left\{f_{k}^{*} \geq 2 f_{k-1}^{*}\right\}}>\lambda\right\} \leq 4 \lambda^{-1}\left\|\sum_{k=1}^{\infty}\left|d \beta_{k}\right|^{2}\right\|_{1}=\frac{4}{\lambda}\|\beta\|_{2}^{2} \leq \mathrm{c}\|f\|_{1} .
$$

In a similar way, since the $\mathrm{E}_{k-1}$ 's are contractive in $L_{3}(\Omega)$, we find

$$
\lambda \sum_{k=1}^{\infty} \mu\left\{\mathrm{E}_{k-1}\left(\left|d \beta_{k}\right| \chi_{\left\{f_{k}^{*} \geq 2 f_{k-1}^{*}\right\}}\right)>\lambda\right\} \leq \mathrm{c}\|f\|_{1} .
$$

The assertion follows from these estimates and the quasi-triangle inequality.

\subsection{Step 2: Proof of the estimate}

$$
\lambda \sum_{k=1}^{\infty} \mu\left\{\left|d \alpha_{k}\right| \chi_{\left\{f_{k}^{*} \geq 2 f_{k-1}^{*}\right\}}>\lambda\right\} \leq \mathrm{c}\|f\|_{1}
$$

Let us fix $\lambda>0$. Since $d \alpha_{k}=d f_{k} \chi_{\left\{f_{k-1}^{*}>\lambda\right\}}$, we have

$$
\lambda \sum_{k=1}^{\infty} \mu\left\{\left|d \alpha_{k}\right| \chi_{\left\{f_{k}^{*} \geq 2 f_{k-1}^{*}\right\}}>\lambda\right\} \leq \lambda \sum_{k=1}^{\infty} \mu\left\{f_{k}^{*} \geq 2 f_{k-1}^{*}>2 \lambda\right\} .
$$


Define $\mathcal{A}: \Omega \rightarrow \mathbb{N} \cup\{\infty\}$ as follows

$$
\mathcal{A}(w)=\sum_{k=1}^{\infty} \mathcal{A}(w, k) \quad \text { with } \quad \mathcal{A}(w, k)= \begin{cases}1 & \text { if } f_{k}^{*}(w) \geq 2 f_{k-1}^{*}(w)>2 \lambda \\ 0 & \text { if not }\end{cases}
$$

According to Doob's maximal inequality, the set of points $w \in \Omega$ with $\mathcal{A}(w)=\infty$ has zero $\mu$-measure. On the other hand, given any integer $s \geq 1$, the set of $w \in \Omega$ such that $\mathcal{A}(w)=s$ is contained in the set where $f^{*}>2^{s} \lambda$. Indeed, note that the condition $f_{k}^{*} \geq 2 f_{k-1}^{*}>2 \lambda$ is satisfied $s$ times. Therefore, by means of Doob's maximal inequality, we deduce

$$
\begin{aligned}
\lambda \sum_{k=1}^{\infty} \mu\left\{f_{k}^{*} \geq 2 f_{k-1}^{*}>2 \lambda\right\} & =\lambda \sum_{k=1}^{\infty} \int_{\Omega} \mathcal{A}(w, k) d \mu(w) \\
& =\lambda \int_{\Omega} \mathcal{A}(w) d \mu(w)=\lambda \sum_{s=1}^{\infty} s \mu(\mathcal{A}=s) \\
& \leq \lambda \sum_{s=1}^{\infty} s \mu\left(f^{*}>2^{s} \lambda\right) \leq\left(\sum_{s=1}^{\infty} s / 2^{s}\right)\|f\|_{1} .
\end{aligned}
$$

This completes the proof since $\sum_{s=1}^{\infty} s / 2^{s}=2$.

\subsection{Step 3: Proof of the last estimate for k-regular martingales}

$$
\lambda \sum_{k=1}^{\infty} \mu\left\{\mathrm{E}_{k-1}\left(\left|d \alpha_{k}\right| \chi_{\left\{f_{k}^{*} \geq 2 f_{k-1}^{*}\right\}}\right)>\lambda\right\} \leq \mathrm{ck}\|f\|_{1}
$$

This is the only estimate where we use the k-regularity of the filtration $A_{1}, A_{2}, \ldots$ Let us introduce some notation. Let us consider a parameter $k>1$. We shall say that the filtration $A_{1}, A_{2}, \ldots$ is $k$-homogeneous if for every $n \geq 1$ and any measurable set $\mathrm{A} \in \mathrm{A}_{n}$ we have

$$
\mu\left\{\operatorname{supp} \mathrm{E}_{n-1}\left(\chi_{\mathrm{A}}\right)\right\} \leq \mathrm{k} \mu(\mathrm{A}) .
$$

We are not aware if this notion appears somewhere in the literature. We now study the relation between $\mathrm{k}_{1}$-regularity and $\mathrm{k}_{2}$-homogeneity. Again we ignore whether or not this result is already known.

Lemma 3.1. Given $1<\mathrm{k}_{1}<\mathrm{k}_{2}<\infty$, we have

$$
\mathrm{k}_{1} \text {-regularity } \Rightarrow \mathrm{k}_{1} \text {-homogeneity } \Rightarrow \mathrm{k}_{2} \text {-regularity. }
$$


Proof. Let us begin with the first implication. Given a positive integer $n \geq 1$ and a measurable set $\mathrm{A} \in \mathrm{A}_{n}$, we known from the assumption on $\mathrm{k}_{1}$-regularity that the inequality below holds

$$
\chi_{\mathrm{A}} \leq \mathrm{k}_{1} \mathrm{E}_{n-1}\left(\chi_{\mathrm{A}}\right) .
$$

Let us assume that

$$
\mu\left\{\operatorname{supp} \mathrm{E}_{n-1}\left(\chi_{\mathrm{A}}\right)\right\}>\mathrm{k}_{1} \mu(\mathrm{A}) .
$$

By the main property of the conditional expectation, we have

$$
\begin{aligned}
\mathrm{k}_{1} \mu(\mathrm{A}) & =\mathrm{k}_{1} \int_{\Omega} \chi_{\mathrm{A}} d \mu=\mathrm{k}_{1} \int_{\Omega} \mathrm{E}_{n-1}\left(\chi_{\mathrm{A}}\right) d \mu \\
& \geq \mathrm{k}_{1} \mu\left\{\operatorname{supp} \mathrm{E}_{n-1}\left(\chi_{\mathrm{A}}\right)\right\} \inf \left\{\mathrm{E}_{n-1}\left(\chi_{\mathrm{A}}\right)(w) \mid w \in \operatorname{supp} \mathrm{E}_{n-1}\left(\chi_{\mathrm{A}}\right)\right\} .
\end{aligned}
$$

In particular, we deduce that

$$
\inf \left\{\mathrm{E}_{n-1}\left(\chi_{\mathrm{A}}\right)(w) \mid w \in \operatorname{supp} \mathrm{E}_{n-1}\left(\chi_{\mathrm{A}}\right)\right\}<1 / \mathrm{k}_{1}
$$

so that the following set in $A_{n-1}$

$$
\mathrm{B}=\left\{0<\mathrm{E}_{n-1}\left(\chi_{\mathrm{A}}\right)<1 / \mathrm{k}_{1}\right\}
$$

has positive $\mu$-measure. If we show $\mu(\mathrm{A} \cap \mathrm{B})>0$, we conclude since $\mathrm{A} \cap \mathrm{B}$ is a subset of $\mathrm{A}$ where (9) fails. Assume that $\mu(\mathrm{A} \cap \mathrm{B})=0$. In that case we have $\chi_{\mathrm{A}} \chi_{\mathrm{B}}=0 \mu$-a.e. and since $\mathrm{B} \in \mathrm{A}_{n-1}$ we conclude

$$
\mathrm{E}_{n-1}\left(\chi_{\mathrm{A}}\right) \chi_{\mathrm{B}}=\mathrm{E}_{n-1}\left(\chi_{\mathrm{A}} \chi_{\mathrm{B}}\right)=0 \quad \mu \text {-a.e. }
$$

However, this contradicts the definition of B. The proof of the first implication is completed.

For the second implication, we have to show that the $\mathrm{A}_{n}$-measurable set $\mathrm{A}=\left\{f_{n}>\mathrm{k}_{2} f_{n-1}\right\}$ has zero $\mu$-measure for any positive martingale $f=\left(f_{n}\right)_{n \geq 1}$ adapted to our filtration. Let us assume that $\mu(\mathrm{A})>0$. Then we consider a $\mathrm{A}_{n}$-measurable function $g$ of the form $g=\sum_{j} \alpha_{j} \chi_{\mathrm{B}_{j}}$, where the sum might have infinitely many terms, the $\mathrm{B}_{j}$ 's are in $\mathrm{A}_{n}$ and such that

$$
g \leq f_{n} \quad \text { and } \quad\left\|g-f_{n}\right\|_{\infty}<\varepsilon
$$

If $w \in \mathrm{A}$, we have

$$
g(w)>f_{n}(w)-\varepsilon>\mathrm{k}_{2} f_{n-1}(w)-\varepsilon \geq \mathrm{k}_{2} \mathrm{E}_{n-1}(g)(w)-\varepsilon .
$$


Therefore it turns out that

$$
\begin{aligned}
\mathrm{A} & \subset\left\{\sum_{j} \alpha_{j} \chi_{\mathrm{B}_{j}}>\mathrm{k}_{2} \sum_{j} \alpha_{j} \mathrm{E}_{n-1}\left(\chi_{\mathrm{B}_{j}}\right)-\varepsilon\right\} \\
& \subset \bigcup_{j \geq 1}\left\{\chi_{\mathrm{B}_{j}}>\mathrm{k}_{2} \mathrm{E}_{n-1}\left(\chi_{\mathrm{B}_{j}}\right)-\varepsilon\right\} .
\end{aligned}
$$

Recalling that $\mu(\mathrm{A})>0$, there must exist some $j_{0} \geq 1$ such that

$$
\mu\left\{\chi_{\mathrm{B}_{j_{0}}}>\mathrm{k}_{2} \mathrm{E}_{n-1}\left(\chi_{\mathrm{B}_{j_{0}}}\right)-\varepsilon\right\}>0 .
$$

We shall denote this set in $\mathrm{A}_{n}$ by $\mathrm{D}_{j_{0}}$. Given $w \in \mathrm{D}_{j_{0}}$, we clearly have

$$
\mathrm{k}_{2} \mathrm{E}_{n-1}\left(\chi_{\mathrm{D}_{j_{0}}}\right)(w)<1+\varepsilon .
$$

On the other hand, by $k_{1}$-homogeneity

$$
\begin{aligned}
\mu\left\{\operatorname{supp} \mathrm{E}_{n-1}\left(\chi_{\mathrm{D}_{j_{0}}}\right)\right\} & \leq \mathrm{k}_{1} \mu\left(\mathrm{D}_{j_{0}}\right)=\mathrm{k}_{1} \int_{\Omega} \mathrm{E}_{n-1}\left(\chi_{\mathrm{D}_{j_{0}}}\right) d \mu \\
& \leq \mathrm{k}_{1} \mu\left\{\operatorname{supp} \mathrm{E}_{n-1}\left(\chi_{\mathrm{D}_{j_{0}}}\right)\right\}\left\|\mathrm{E}_{n-1}\left(\chi_{\mathrm{D}_{j_{0}}}\right)\right\|_{\infty} .
\end{aligned}
$$

This means that $\left\|\mathrm{E}_{n-1}\left(\chi_{\mathrm{D}_{j_{0}}}\right)\right\|_{\infty} \geq 1 / \mathrm{k}_{1}$, hence

$$
\mu\left(\mathrm{R}_{\delta}\right)>0 \quad \text { with } \quad \mathrm{R}_{\delta}=\left\{\mathrm{E}_{n-1}\left(\chi_{\mathrm{D}_{j_{0}}}\right)>1 / \mathrm{k}_{1}-\delta\right\} \quad \text { for every } \delta>0 .
$$

Now we observe that for any $w \in \mathrm{D}_{j_{0}} \cap \mathrm{R}_{\delta}$ we obtain

$$
\frac{\mathrm{k}_{2}}{\mathrm{k}_{1}}-\delta \mathrm{k}_{2}<\mathrm{k}_{2} \mathrm{E}_{n-1}\left(\chi_{\mathrm{D}_{j_{0}}}\right)(w)<1+\varepsilon .
$$

Therefore, since we are assuming that $\mathrm{k}_{1}<\mathrm{k}_{2}$, we may take $\delta$ and $\varepsilon$ small enough so that the relation above provides the desired contradiction as far as we show that $\mu\left(D_{j_{0}} \cap R_{\delta}\right)>0$. However, this follows as in the first part of the proof. Indeed, if not we would have $\chi_{\mathrm{D}_{j_{0}}} \chi_{\mathrm{R}_{\delta}}=0 \mu$-a.e. Thus since $\mathrm{R}_{\delta} \in \mathrm{A}_{n-1}$ we should conclude that $\mathrm{E}_{n-1}\left(\chi_{\mathrm{D}_{j_{0}}}\right) \chi_{\mathrm{R}_{\delta}}=0 \mu$-a.e., which contradicts the definition of $\mathrm{R}_{\delta}$.

Now it is straightforward to finish the proof of Theorem B. Namely, it remains to prove inequality (8). However, since we assume that $f$ is a positive martingale, we have by k-regularity that $d f_{k} \leq(\mathrm{k}-1) f_{k-1}$. Therefore, assuming k-regularity and according to the first half of Lemma 3.1 we obtain

$$
\begin{aligned}
\lambda \sum_{k=1}^{\infty} \mu\left\{\mathrm{E}_{k-1}\left(\left|d \alpha_{k}\right| \chi_{\left\{f_{k}^{*} \geq 2 f_{k-1}^{*}\right\}}\right)\right. & >\lambda\} \leq \lambda \sum_{k=1}^{\infty} \mu\left\{\mathrm{E}_{k-1}\left(\chi_{\left\{f_{k}^{*} \geq 2 f_{k-1}^{*}>2 \lambda\right\}}\right)>0\right\} \\
& \leq \mathrm{k} \lambda \sum_{k=1}^{\infty} \mu\left\{f_{k}^{*} \geq 2 f_{k-1}^{*}>2 \lambda\right\} \leq 2 \mathrm{k}\|f\|_{1},
\end{aligned}
$$

where the last inequality follows by Step 2. Theorem B is proved. 


\subsection{Further remarks}

We conclude this section by analyzing Theorem B in some detail.

If we do not care about its validity for non-regular martingales, a much simpler proof is available. The idea is that regular martingales are previsible, see e.g. Proposition 2.19 in [22]. This can be used to show that the only relevant part in $\left(\mathrm{WB}_{2}\right)$ for regular martingales is the term associated to the conditional square function.

A simpler proof of Theorem B. Let $f=\left(f_{1}, f_{2}, \ldots\right)$ be a bounded positive martingale in $L_{1}(\Omega)$ adapted to a k-regular filtration. Then we may consider the Davis type decomposition $f=g+h$ with martingale differences given by

$$
\begin{aligned}
d g_{k} & =d f_{k} \chi_{\left\{f_{k} \leq \mathrm{k} f_{k-1}\right\}}-\mathrm{E}_{k-1}\left(d f_{k} \chi_{\left\{f_{k} \leq \mathrm{k} f_{k-1}\right\}}\right), \\
d h_{k} & =d f_{k} \chi_{\left\{f_{k}>\mathrm{k} f_{k-1}\right\}}-\mathrm{E}_{k-1}\left(d f_{k} \chi_{\left\{f_{k}>\mathrm{k} f_{k-1}\right\}}\right) .
\end{aligned}
$$

By k-regularity, the only non-zero martingale difference in $h$ is

$$
d h_{1}=f_{1} .
$$

Thus, the second term in $\left(\mathrm{WB}_{2}\right)$ is trivially controlled by $\|f\|_{1}$. On the other hand, the first term in $\left(\mathrm{WB}_{2}\right)$ can be estimated as in Step 1 of the proof of Theorem A, with the only difference that we obtain the constant ck instead of c. The proof is complete.

Remark 3.2. The proof given above shows that in the k-regular case it suffices to consider the conditional term and ignore the diagonal one. As it was justified in Remark 8.3 of [2], this is only possible under the assumption of regularity.

As we already mentioned in the Introduction, martingale inequalities where a martingale decomposition is involved arise very naturally in the noncommutative setting, mainly due to the row/column nature of the corresponding martingale Hardy spaces.

Among many other papers, we refer the reader to $[14,16,23]$ for some illustrations of this phenomenon. However, not requiring the decompositions to be martingale decompositions, it is sometimes simpler to obtain the corresponding inequality $[19,20]$.

Our first proof of Theorem B goes a little further and produces the following result in this line. 
Corollary C. Let $f=\left(f_{1}, f_{2}, \ldots\right)$ be a bounded martingale in $L_{1}(\Omega)$. Then, we can decompose each $f_{n}$ as a sum $f_{n}=g_{n}+h_{n}$ of two functions (nonnecessarily martingales) adapted to the same filtration and satisfying the following inequality with an absolute constant c

$$
\left\|\left(\sum_{k=1}^{\infty} \mathrm{E}_{k-1}\left(\left|d g_{k}\right|^{2}\right)\right)^{\frac{1}{2}}\right\|_{L_{1, \infty}(\Omega)}+\left\|\sum_{k=1}^{\infty} \delta_{k} \otimes d h_{k}\right\|_{L_{1, \infty}\left(\Omega_{\oplus \infty}\right)} \leq \mathrm{c}\|f\|_{1} .
$$

Proof. If we consider the decomposition

$$
\begin{aligned}
d g_{k} & =d f_{k} \chi_{\left\{f_{k}^{*}<2 f_{k-1}^{*}\right\}}, \\
d h_{k} & =d f_{k} \chi_{\left\{f_{k}^{*} \geq 2 f_{k-1}^{*}\right\}}
\end{aligned}
$$

it follows from our first proof of Theorem B since (8) is not needed.

Namely, we can drop the k-regularity assumption as far as we do not require to have a decomposition of $f$ into two martingales. We have proved Theorem B under the assumption of k-regularity or avoiding martingale decompositions as in Corollary C. However, it is still open to decide whether Theorem B holds for arbitrary martingales. Let us state this problem for the interested reader.

Problem 3.3. Let $f=\left(f_{1}, f_{2}, \ldots\right)$ be a bounded martingale in $L_{1}(\Omega)$. Is there a decomposition of $f$ as a sum $f=g+h$ of two martingales adapted to the same filtration and satisfying the following inequality with an absolute constant $\mathrm{c}$ ?

$$
\left\|\left(\sum_{k=1}^{\infty} \mathrm{E}_{k-1}\left(\left|d g_{k}\right|^{2}\right)\right)^{\frac{1}{2}}\right\|_{L_{1, \infty}(\Omega)}+\left\|\sum_{k=1}^{\infty} \delta_{k} \otimes d h_{k}\right\|_{L_{1, \infty}\left(\Omega_{\oplus \infty}\right)} \leq \mathrm{c}\|f\|_{1} .
$$

\section{Applications and Comments}

In this section, we obtain some applications of Theorems A and B. At the very end, we analyze their relation to the theory of noncommutative martingales. The reader who is not familiar with the theory of noncommutative martingales can skip this part, which is not related to the main purpose of this paper. Let us begin by studying the implications of Theorem A in Davis decomposition.

\subsection{On the classical Davis decomposition}

Besides its clear relation with Burkholder's martingale inequality, Theorem A can also be understood as a weak type estimate which generalizes the 
known properties of Davis decomposition. In other words, recalling the second estimate in Davis decomposition (1)

$$
\left\|\sum_{k=1}^{\infty}\left|d h_{k}\right|\right\|_{p} \leq \mathrm{c} p\left\|f^{*}\right\|_{p} \leq \mathrm{c} \frac{p^{2}}{p-1}\|f\|_{p} \quad \text { for } \quad 1<p<\infty,
$$

the inequality

$$
\left\|\sum_{k=1}^{\infty}\left|d h_{k}\right|\right\|_{1, \infty} \leq \mathrm{c}\|f\|_{1}
$$

can be regarded as the associated weak type inequality for $p=1$. This inequality was justified in Steps 2 and 3 of our proof of Theorem A. On the other hand, in a less explicit way, the estimate for the first term in $\left(\mathrm{WB}_{1}\right)$ can also be understood as an extension of the known estimates for $g$ in Davis decomposition.

\subsection{Real interpolation}

One of the first applications of our results that comes to mind is reproving Burkholder's inequality by real interpolation and duality. This is even possible starting from Corollary C, as showed in [20]. This alternative proof has given rise to the optimal constants for the noncommutative Burkholder inequality [12]. It can be easily checked that the constants obtained in [20] are still optimal in the commutative case as $p \rightarrow 1$ but not as $p \rightarrow \infty$, as it follows from Hitczenko's results [8].

We should also point out that, in contrast with the previous paragraph (where Theorem A came into scene), Theorem B is the right result to obtain Burkholder's inequality using real interpolation. Indeed, if we want to obtain $\left(\mathrm{B}_{p}^{-}\right)$from Theorem $\mathrm{B}$, we just need the well-known isomorphism

$$
\left[L_{1, \infty}\left(\Omega_{\oplus \infty}\right), L_{2}\left(\Omega_{\oplus \infty}\right)\right]_{\theta, p} \sim_{c_{p}} L_{p}\left(\Omega_{\oplus \infty}\right) \quad \text { with } \quad c_{p} \sim p / p-1 .
$$

On the contrary, Theorem A would require something different.

Question 4.1. Is is true that

$$
\left[L_{1, \infty}\left(\Omega ; \ell_{1}\right), L_{2}\left(\Omega ; \ell_{2}\right)\right]_{\theta, p} \sim_{\mathrm{c}_{p}} L_{p}\left(\Omega ; \ell_{p}\right) ?
$$

This question is reminiscent of other natural questions with regard to extending Marcinkiewicz interpolation theorem to more general situations: bilinear operators, changes of weights or, in our case, vector-valued function spaces... This particular question seemed quite unclear and finally Michael 
Cwikel showed to me [4] that the result conjectured above is false. Let us include Cwikel's argument for completeness. Let us define

$$
\mathcal{A}_{0}=L_{1, \infty}\left(\Omega ; \ell_{1}\right) \quad \text { and } \quad \mathcal{A}_{1}=L_{2}\left(\Omega ; \ell_{2}\right)
$$

It is clear that $\mathcal{A}_{0}$ contains $L_{1}\left(\Omega_{\oplus_{\infty}}\right)$, so that the inclusion below holds

$$
L_{p}\left(\Omega ; \ell_{p}\right)=L_{p}\left(\Omega_{\oplus \infty}\right) \subset\left[\mathcal{A}_{0}, \mathcal{A}_{1}\right]_{\theta, p} .
$$

However, the reverse inclusion fails. Following Cwikel's argument we show that the even smaller space $\mathcal{A}_{0} \cap \mathcal{A}_{1}$ is not contained in $L_{p}\left(\Omega ; \ell_{p}\right)$. To that aim we take $\Omega$ to be the unit interval equipped with the Lebesgue measure. We will represent elements of $\mathcal{A}_{0} \cap \mathcal{A}_{1}$ as step functions $f:[0,1] \times \mathbb{R}_{+} \rightarrow \mathbb{C}$ of two variables $(w, z)$. Of course, we need to assume that for each constant $w$, the function $f(w, \cdot)$ is a constant function of $z$ on the interval $(n-1, n]$ for each positive integer $n$. Let us consider the following sets in the plane

$$
\mathcal{S}=\bigcup_{k \geq 1} \mathcal{S}_{k} \quad \text { with } \quad \mathcal{S}_{k}=\{0 \leq w \leq 1 / k \text { and } k-1<z \leq k\}
$$

The set $\mathcal{S}$ is a discretized version of the region where $0<z \leq 1 / w$. Let $f_{1}$ be the characteristic function of $\mathcal{S}$. We claim that, under the representation considered above, $f_{1}$ is an element of $\mathcal{A}_{0}$. Indeed, we have

$$
\left\|f_{1}\right\|_{\mathcal{A}_{0}}=\sup _{\lambda>0} \lambda \mu\left\{\sum_{k}\left|f_{1}(w, k)\right|>\lambda\right\}=1<\infty
$$

Now, let $\alpha>0$ be a positive number and let

$$
f_{2}(w, k)=\frac{\chi_{\mathcal{S}_{k}}(w)}{(1+\log k)^{\alpha}} \quad \text { for } \quad k \geq 1
$$

Since $f_{2} \leq f_{1}$, it follows that $f_{2} \in \mathcal{A}_{0}$. Moreover, we have

$$
\left\|f_{2}\right\|_{L_{p}\left(\Omega ; \ell_{p}\right)}=\left(\sum_{k}\left\|\frac{\chi_{\mathcal{S}_{k}}(w)}{(1+\log k)^{\alpha}}\right\|_{p}^{p}\right)^{\frac{1}{p}}=\left(\sum_{k} \frac{1}{k(1+\log k)^{p \alpha}}\right)^{\frac{1}{p}}
$$

so that $f_{2} \in L_{p}\left(\Omega, \ell_{p}\right)$ if and only if $p \alpha>1$. Therefore, given any $1<p<2$ we may choose $\alpha$ so that $p \alpha<1<2 \alpha$. In this case we obtain that

$$
f_{2} \in \mathcal{A}_{0} \cap \mathcal{A}_{1} \backslash L_{p}\left(\Omega ; \ell_{p}\right)
$$




\subsection{A noncommutative Davis type decomposition}

In this paragraph we present a useful way to generalize the martingale differences $d g_{k}$ and $d h_{k}$ in Davis martingale decomposition to the noncommutative setting. We shall assume certain familiarity with the theory of noncommutative martingale inequalities. We begin with the trivial identity

$$
\begin{aligned}
\left\{f_{k}^{*}\right. & \left.<2 f_{k-1}^{*}\right\}=\bigcup_{\lambda>0}\left\{\frac{\lambda}{2}<f_{k-1}^{*} \leq \lambda\right\} \cap\left\{\frac{\lambda}{2}<f_{k}^{*} \leq \lambda\right\} \\
& =\bigcup_{\lambda>0}\left(\left\{f_{k-1}^{*} \leq \lambda\right\} \backslash\left\{f_{k-1}^{*} \leq \frac{\lambda}{2}\right\}\right) \cap\left(\left\{f_{k}^{*} \leq \lambda\right\} \backslash\left\{f_{k}^{*} \leq \frac{\lambda}{2}\right\}\right) .
\end{aligned}
$$

One important lack in noncommutative martingales is the absence of stopping times or maximal functions. In the case of maximal functions, there exist two natural substitutes. Roughly speaking, we use a construction due to Cuculescu [3] when dealing with weak type inequalities, while for strong inequalities the right notion was formulated by Junge in [10]. We shall use here Cuculescu's construction. In other words, for any $\lambda>0$ we can construct a sequence of projections $q_{1}(\lambda), q_{2}(\lambda), \ldots$ which play the role of the sets $\left\{f_{k}^{*} \leq \lambda\right\}$ for $k=1,2, \ldots$ Namely, the sequence of $q_{k}(\lambda)$ 's is adapted for all $\lambda>0$ and satisfies an analogue of the weak type $(1,1)$ Doob's maximal inequality, see $[3,15,18]$ for more details. There is however one natural property which is not satisfied by Cuculescu's projections. In contrast with the classical case, where we have $\left\{f_{k}^{*} \leq \lambda_{1}\right\} \subset\left\{f_{k}^{*} \leq \lambda_{2}\right\}$ whenever $\lambda_{1}<\lambda_{2}$, it is no longer true that $q_{k}\left(\lambda_{1}\right)$ is a subprojection of $q_{k}\left(\lambda_{2}\right)$. This is solved by defining the projections

$$
\pi_{k}(\lambda)=\bigwedge_{\xi \geq \lambda} q_{k}(\xi) \sim \bigcap_{\xi \geq \lambda}\left\{f_{k}^{*} \leq \xi\right\}=\left\{f_{k}^{*} \leq \lambda\right\} .
$$

However, for some technical reasons like commuting properties of the resulting projections, it is better to consider countable families of $q_{k}$ 's. More concretely, we consider dyadic $\lambda$ 's of the form $\lambda=2^{j}$ and define

$$
\pi_{k}(\lambda)=\bigwedge_{s=0}^{\infty} q_{k}\left(2^{j+s}\right) \sim\left\{f_{k}^{*} \leq 2^{j}\right\}
$$

This gives rise to the following approximation

$$
\begin{aligned}
\left\{f_{k}^{*}<2 f_{k-1}^{*}\right\} & \sim \bigvee_{j=0}^{\infty}\left(\pi_{k-1}\left(2^{j}\right)-\pi_{k-1}\left(2^{j-1}\right)\right) \wedge\left(\pi_{k}\left(2^{j}\right)-\pi_{k}\left(2^{j-1}\right)\right) \\
& =\sum_{j=0}^{\infty}\left(\pi_{k-1}\left(2^{j}\right)-\pi_{k-1}\left(2^{j-1}\right)\right) \wedge\left(\pi_{k}\left(2^{j}\right)-\pi_{k}\left(2^{j-1}\right)\right)
\end{aligned}
$$


where the last identity follows by pairwise orthogonality. Let us observe in passing that the information lost in the process of taking only dyadic $\lambda$ 's is not relevant since our proofs of Theorems A and B still hold when replacing the sets $\left\{f_{k}^{*}<2 f_{k-1}^{*}\right\}$ by the smaller ones

$$
\bigcup_{j=0}^{\infty}\left\{2^{j-1}<f_{k-1}^{*} \leq f_{k}^{*} \leq 2^{j}\right\}
$$

We have already rewritten the Davis sets $\left\{f_{k}^{*}<2 f_{k-1}^{*}\right\}$ in a way that works in the noncommutative setting. Of course, this gives rise to a Davis type decomposition for noncommutative martingales. Although not mentioned by Randrianantoanina, this might be a good motivation for the decomposition used in [20]. Indeed, although the decomposition in [20] is not made of martingale differences, the same idea is used there in a very indirect way. More concretely, the projections

$$
p_{j, k}=\bigwedge_{s=j}^{\infty} q_{k}\left(2^{s}\right)-\bigwedge_{s=j-1}^{\infty} q_{k}\left(2^{s}\right)=\pi_{k}\left(2^{j}\right)-\pi_{k}\left(2^{j-1}\right)
$$

as well as $p_{j, k-1} p_{j, k}$ are important key tools in [20]. Nevertheless, it is also worthy of mention that the decomposition used there is not exactly the translation (via the transformations described in this paragraph) of this paper. Namely, an extra nonsymmetric row/column partition is needed in the noncommutative case.

Finally, we observe that the noncommutative Davis decomposition presented here is constructed with the aim to interact with Cuculescu's construction. In other words, according to the philosophy mentioned above, this decomposition should be the right one when dealing with weak type inequalities. For strong inequalities, we should work with Junge's approach [10], but we still do not know how to obtain the right maximal operators and Davis decomposition is still unclear.

\subsection{Related results}

This paper is strongly motivated by problems and results from noncommutative probability. We conclude by giving some references (not included in the Introduction) related to this paper. The optimal growth of the relevant constants in several noncommutative martingale inequalities can be found in [13]. Gundy's decomposition of noncommutative martingales was obtained in the recent paper [15]. There also exists free analogs of generalized Khintchine and Rosenthal inequalities, see [11] and the references therein. 


\section{References}

[1] Burkholder, D. L.: Distribution function inequalities for martingales. Ann. Probab. 1 (1973), 19-42.

[2] Burkholder, D. L. And Gundy, R. F.: Extrapolation and interpolation of quasi-linear operators on martingales. Acta Math. 124 (1970), 249-304.

[3] Cuculescu, I.: Martingales on von Neumann algebras. J. Multivariate Anal. 1 (1971), 17-27.

[4] Cwikel, M.: Personal communication.

[5] Davis, B.: On the integrability of the martingale square function. Israel $J$. Math. 8 (1970), 187-190.

[6] Garsia, A. M.: Martingale inequalities: Seminar notes on recent progress. Mathematics Lecture Notes Series. W. A. Benjamin, Inc., Reading, Mass.London-Amsterdam, 1973.

[7] Gundy, R. F.: A decomposition for $L^{1}$-bounded martingales. Ann. Math. Statist. 39 (1968), 134-138.

[8] Hitczenko, P.: Best constants in martingale version of Rosenthal's inequality. Ann. Probab. 18 (1990), 1656-1668.

[9] Johnson, W. B., Schechtman, G. and Zinn, J.: Best constants in moment inequalities for linear combinations of independent and exchangeable random variables. Ann. Probab. 13 (1985), 234-253.

[10] Junge, M.: Doob's inequality for non-commutative martingales. J. Reine Angew. Math. 549 (2002), 149-190.

[11] Junge, M., Parcet, J. And Xu, Q.: Rosenthal type inequalities for free chaos. Ann. Probab. 35 (2007), no. 4, 1374-1437.

[12] Junge, M. And Xu, Q.: Noncommutative Burkholder/Rosenthal inequalities. Ann. Probab. 31 (2003), 948-995.

[13] Junge, M. And Xu, Q.: On the best constants in some non-commutative martingale inequalities. Bull. London Math. Soc. 37 (2005), 243-253.

[14] Lust-Piquard, F. And Pisier, G.: Non-commutative Khintchine and Paley inequalities. Ark. Mat. 29 (1991), 241-260.

[15] Parcet, J. and Randrianantoanina, N.: Gundy's decomposition for non-commutative martingales and applications. Proc. London Math. Soc. (3) 93 (2006), 227-252.

[16] Pisier, G. AND Xu, Q.: Non-commutative martingale inequalities. Comm. Math. Phys. 189 (1997), 667-698.

[17] Pisier, G. And Xu, Q.: Non-commutative $L_{p}$-spaces. In Handbook of the Geometry of Banach Spaces, II, 1459-1517. North-Holland, Amsterdam, 2003.

[18] Randrianantoanina, N.: Non-commutative martingale transforms. J. Funct. Anal. 194 (2002), 181-212. 
[19] Randrianantoanina, N.: Square function inequalities for noncommutative martingales. Israel J. Math. 140 (2004), 333-365.

[20] Randrianantoanina, N.: Conditioned square functions for noncommutative martingales. Ann. Probab. 35 (2007), no. 3, 1039-1070.

[21] Rosenthal, H. P.: On the subspaces of $L^{p}(p>2)$ spanned by sequences of independent random variables. Israel J. Math. 8 (1970), 273-303.

[22] Weisz, F.: Martingale Hardy spaces and its applications in Fourier analysis. Lecture Notes in Mathematics, 1568. Springer-Verlag, Berlin, 1994.

[23] XU, Q.: Recent development on non-commutative martingale inequalities. In Functional space theory and its applications, 283-314. Proceedings of International Conference \& 13th Academic Symposium in China. Ed. Research Information Ltd UK. Wuhan, 2003.

Recibido: 15 de septiembre de 2005

Revisado: 3 de mayo de 2006

Javier Parcet

Departamento de Matemáticas

Instituto de Matemáticas y Física Fundamental Consejo Superior de Investigaciones Científicas

C/ Serrano 121, 28006 Madrid. Spain javier.parcet@uam.es

Partially supported by "Programa Ramón y Cajal 2005" and Projects MTM2004/00678, MTM2007-60952 and CCG06-UAM/ESP-0286, Spain. 